\title{
Reactivity of
}

\section{2-Cyano-N-(4-(1-Methyl-1H-benzo[d]imidazol-2-yl)-3-(M ethylthio)-1-Phenyl-1H-Pyrazol-5-yl)Acetamide: A Facile Synthesis of Pyrazole, Thiazole, 1,3,4-Thiadiazole and Polysubstituted Thiophene Derivatives}

\author{
Mohamed A. Khalil, Samia M. Sayed, Mohamed A. Ras lan* \\ Chemistry Department, Faculty of Science, Aswan University, 81528 Aswan, Egypt
}

\begin{abstract}
Treatment of 2-cyano-N-(4-(1-methyl-1H-benzo[d]imidazol-2-yl)-3-(methylthio)-1-phenyl-1H-pyrazol-5-yl) acetamide (4) with phenyl isothiocyanate gave the thiole derivative (6) which on treatment with hydrazonyl chlorides (7a-c) furnished 1,3,4-thiadiazole derivatives (9a-c). Reaction of cyanoacetamide derivative (4) with active methylene reagents such as malononitrile or ethyl cyanoacetate and elemental sulfur afforded the corresponding polysubstituted thiophene derivatives $(18 \mathrm{a}, \mathrm{b})$. Reaction of cyanoacetamide derivative (4) with benzaldehyde yielded the phenylmethylidene derivative (21). The latter showed interesting reactivity towards cyanomethylene reagent and hydrazine derivatives afforded pyrane $(22 \mathrm{a}, \mathrm{b})$ and pyrazole $(25 \mathrm{a}, \mathrm{b})$ derivatives.
\end{abstract}

Keywords Cyanoacetamide, Active Methylene, Hydrazonyl Halides , $\alpha$-halo-carbonyl Compounds

\section{Introduction}

Several pyrazole derivatives are important pharmaceutical, they have been found to possess diverse biological activities [1-6]. They are also acknowledged for their anticancer[7-11], antipyretic[12], anti-inflammatory[13], antimicrobial activit ies[14-16], antiviral[17], tranquillizing [18], antihypertensi ve[19], antidepressant[20], antiarrhythmi [21], psychoanalep tic[22], anticonvulsant[23] and antidiabetic activities [24]. Moreover, the thiazole derivative has received a great deal of attention due to their pharmacological importance. Thiazoles were reported to possess antimic robial[25-28], analgesic[29], anti-inflammatory[30], anticonvulsant[31], card iotonic[32], anticancer[33,34], antitubercular[35] and anthelmintic[36] activites. Antimicrbial activities of some substituted thiazoles are well established because it posses $(\mathrm{S}-\mathrm{C}=\mathrm{N})$ toxophoric unit. Thiazoles have enhanced lipid solubility with hydrophilicity. Also, thiazoles are easily metabolized by routine biochemical reactions and are non-carcinogenic in nature[37]. many thiophene containing annulated compoun ds, exhibite bio logical activities[38,39].

In addition, benzimidazole has been an important

* Corresponding author:

raslanma47@yahoo.com (Mohamed A. Raslan)

Published online at http://journal.sapub.org/ajoc

Copyright (C) 2012 Scientific \& Academic Publishing. All Rights Reserved pharmacophore and privileged structure in medicinal chemistry[40] encompassing a diverse rang of biological activities including antiarrhythmic, antiulcer, antih istamine, antifungal, antiviral and cytotoxic ity [41]. Furthermore, 1,3,4 -thiadia zoles were reported as highly anti- inflammatory[42], antimicrobial[43], anticonvulsant[44] and anticancer[45]. agents.

Cyanoacetamides are highly reactive compounds. They are extensively utilized as reactants or reaction intermediate since the carbonyl and the cyano functions of these compounds are suitably situated to enable reactions with common bidentate reagents to form a variety of heterocyclic compounds. Moreover function, the active hydrogen on C-2 of these compounds can take part in a variety of condensation and substitution reactions. The synthes is of cyanoacetamides may be carried out in several ways [46, 47]. Cyanoacetyl pyrazole is very handly and cheap cyanoacetylation reagent It was success fully applied for the synthesis of various $\mathrm{N}$-alky and $\mathrm{N}$-aryl cyanoecatamides. The latter are poly functional compounds possessing both electrophlic and nucleophlilic properties. Cyanoacetamide can be useful for the synthesis of three-six membered rings [48].

In connection with our previous studies [49-58] and in view of utilizing the cyanoacetamide as highly versatile intermediates for the construction of functionlized pyrazole, thiazole, 1,3,4-thiadiazole and polysubstituted thiophene 
derivatives of expected potential biological activity and excellent pharmacology encouraged us to synthesis novel entitled derivatives.

\section{Chemistry}

Treatment of 2-(1-methyl-1- $H$-benzo [d]imidazol-2-yl)-3, 3-bis-(methylthio)acrylonitrile (2) with phenylhydrazine affords the target4-(1-methyl-1H-benzo[d]imidazol-2yl)-3-(methylthio)-1-phenyl-1 $H$-pyrazol-5-amine (3). The structure of (3) was established and confirmed as the reaction product on the basis of their elemental analys is and spectral data. The IR spectrum showed absorption band in the region $3285 \mathrm{~cm}^{-1}$ assignable for $\mathrm{NH}_{2}$, in addition to disappearance of cyano function signal. Its ${ }^{1} \mathrm{H}$ NMR spectrum revealed the presence of singlet signals at $\delta 2.62 \mathrm{ppm}, \delta 3.98 \mathrm{ppm}$ and $\delta$ $5.72 \mathrm{ppm}$ assignable to the $\mathrm{SCH}_{3}, \mathrm{~N}-\mathrm{CH}_{3}$ and $\mathrm{NH}_{2}$ protons, respectively. Its mass spectrum showed a molecular ion peak at $\mathrm{m} / \mathrm{z}=335$ corresponding to a molecular formula $\mathrm{C}_{18} \mathrm{H}_{17} \mathrm{~N}_{5} \mathrm{~S}$. (Scheme 1)

Treatment of 4-(1-methyl-1H-benzo[d]imidazol-2-yl)3-(methylthio)-1-phenyl-1H-pyrazo 1-5-amine (3) with (3,5dimethy-1H-pyrazol -1-yl)-3-oxopropanenitrile as cyanoac etylation reagent in dry toluene afforded 2-cyano-N-(4(1-methy 1-1H-benzo[d]imidazol-2-yl)-3-(methylthio)-1-phe nyl-1 $H$-pyrazol-5-yl)acetamide (4). The structure of (4) was established on the basis of spectral data. The IR spectrum revealed absorption band at $3215 \mathrm{~cm}^{-1}$ for the $\mathrm{NH}$ group, sharp band at $2218 \mathrm{~cm}^{-1}$ for the cyano function and strong band at $1658 \mathrm{~cm}^{-1}$ for carbonyl group. Its ${ }^{1} \mathrm{H}$ NMR spectrum revealed the presence of singlet signals at $\delta 4.12 \mathrm{ppm}$ and $\delta$ $10.65 \mathrm{ppm}$ assignable to the methylene and $\mathrm{NH}$ portons, respectively. Its mass spectrum showed a molecular ion peak at $\mathrm{m} / \mathrm{z}=402$ corresponding to a molecular formula $\mathrm{C}_{21} \mathrm{H}_{18} \mathrm{~N}_{6} \mathrm{OS}$. The reactivity of 2-cyano-N-(4-(1-methyl$1 H$-benzo[d]imidazo 1-2-yl)-3-(methylthio)-1-phenyl-1 $H$-pyr -azol-5-yl) acetamide (4) to give pyrazole, thiazole, thiophene and 1,3,4-thiadiazole derivatives was investigated. Thus, reaction of (4) with phenyl isothiocyanate in DMF and potassium hydroxide solution afforded the corresponding non-isolable enaminonitrile (5) which converted $(\mathbf{6 A})$ or $(\mathbf{6 B})$ form upon treatment with dilute hydrochloric acid (scheme 2). ${ }^{1} \mathrm{H}$ NMR spectrum of the reaction product (6) was free from any aliphatic protons which mean that compound (6) is solely present in $(\boldsymbol{6} \boldsymbol{B})$ form instead of its tautomer $(\boldsymbol{6} \boldsymbol{A})$.

The IR spectrum of (6) revealed absorption bands at 3362 $\mathrm{cm}^{-1}, 3215 \mathrm{~cm}^{-1}$ for the $\mathrm{NH}$ groups, absorption at $2206 \mathrm{~cm}^{-1}$ for the cyano function and absorption band at $1653 \mathrm{~cm}^{-1}$ for carbonyl group. Its ${ }^{1} \mathrm{H}$ NMR spectrum revealed the presence of two singlet signal at $\delta 9.85 \mathrm{ppm}, \delta 10.65 \mathrm{ppm}$ assignable to the $\mathrm{NH}$ protons and singlet signal at $\delta 13.84 \mathrm{ppm}$ assignable to the $\mathrm{SH}$ group. Its mass spectrum showed a molecular ion peak at $\mathrm{m} / \mathrm{z}=537$ corresponding to a mo lecular formula $\left(\mathrm{C}_{28} \mathrm{H}_{23} \mathrm{~N}_{7} \mathrm{OS}_{2}\right)$.

Compound (6) is a versatile multifunctional reagent and its reactivity towards hydrazonyl chloride (7a-c) was studied.
Thus, reaction of compound (6) withC-phenyl-Nphenylhydrazonyl chloride (7a) in refluxing ethanol solution containing triethylamine as basic catalyst afforded solely the corresponding 1,3,4-thiadiazole derivatives (9a-c). Formation of the latter structures is assumed proceed via elimination of aniline molecule from the non-isolable intermediate (8) as outlined in scheme 2. The 1, 3, 4-thiadiazo le structure derivative (9a-c) was confirmed from the elemental analyses and spectral data of the isolated product. The IR spectrum of (9a) revealed absorption band at $2208 \mathrm{c} \mathrm{m}^{-1}$ assignable to cyano function and absorption band at $1662 \mathrm{~cm}^{-1}$ assignable to carbonyl group. Its ${ }^{1} \mathrm{H}$ NMR spectrum spectrum revealed singlet signal for $\mathrm{NH}$ at $\delta 9.98$ $\mathrm{ppm}$ in addition to multiplet signal for aro matic protons at $\delta$ 7.37-8.12 ppm. Its mass spectrum showed a molecular ion peak at $\mathrm{m} / \mathrm{z}=638$ corresponding to a molecular formula $\left(\mathrm{C}_{35} \mathrm{H}_{26} \mathrm{~N}_{8} \mathrm{OS}_{2}\right)$.

In similar manner compound (6) reacted with equimolar amounts of C-acetyl-N-phenylhydrazonyl chloride (7b) and C-ethyl carboxylate-N-phenylhydrazonyl chloride (7c) frunished in each case one isolable product (as tested by TLC analyses) which have the 1,3,4-thiad iazole structures $(\mathbf{9 b , c})$, respectively based on their elemental and spectral analyses (scheme 2).

On the other hand we investigate the reactivity of non-isolable enaminonitrile (5) with $\alpha$-halocarbonyl compounds (10a-c). These reactions may be lead to formation of either thiazole or thiophene systems or both depending on the reaction conditions and the nature of the $\alpha$-halocarbonyl reagent[59]. Thus, reaction of (5) in situ with $\alpha$-halo-carbonyl compounds such as bromoacetone, ethyl bromoacetate and 1-(benzothiazol-2-yl)-2-bromoethanone (10a-c), respectively resulted in the formation of single product for wh ich the thiazole (12), (13 b,c) or thiophene (14) structures, can be assumed. However the elemental and spectral data of the reaction product were incomplete accordance with the thiazole derivatives (12) and (13b,c). The IR spectrum for (12) displayed stretching absorption band at $3347 \mathrm{~cm}^{-1}$ and $2223 \mathrm{~cm}^{-1}$ for the NH and cyano function, respectively and two carbonyl absorption band at 1685 and $1648 \mathrm{~cm}^{-1}$. Its ${ }^{1} \mathrm{H}$ NMR spectrum displayed singlet signals at $\delta 10.31 \mathrm{ppm}$ assignable to the $\mathrm{NH}$ porton and singlet signal at $\delta 3.73 \mathrm{ppm}$ assignable to the methylene protons. The IR spectrum of (13 b) displayed stretching absorption band at $3218 \mathrm{~cm}^{-1}$ and $2204 \mathrm{~cm}^{-1}$ for the NH and cyano function. Its ${ }^{1} \mathrm{H}$ NMR spectrum revealed new signal at $\delta 1.72 \mathrm{ppm}$ for methyl portons and $\delta 6.93 \mathrm{ppm}$ assignable for thiazole $=\mathrm{CH}$ proton and singlet signal at $\delta 10.53 \mathrm{ppm}$ assignable to the $\mathrm{NH}$ group. Its mass spectrum showed a molecular ion peak at $\mathrm{m} / \mathrm{z}=575$ corresponding to a mo lecular formu la $\left(\mathrm{C}_{31} \mathrm{H}_{25} \mathrm{~N}_{7} \mathrm{OS}_{2}\right)$.

The mass spectrum of compounds (12) and $(\mathbf{1 3 b}, \mathbf{c})$ showed a molecular ion peak at $\mathrm{m} / \mathrm{z}=577,575$ and 694 corresponding to the molecular formula $\left(\mathrm{C}_{30} \mathrm{H}_{23} \mathrm{~N}_{7} \mathrm{O}_{2} \mathrm{~S}_{2}\right)$, $\left(\mathrm{C}_{31} \mathrm{H}_{25} \mathrm{~N}_{7} \mathrm{OS}_{2}\right)$, and $\left(\mathrm{C}_{37} \mathrm{H}_{26} \mathrm{~N}_{8} \mathrm{OS}_{3}\right)$, respectively. (scheme 3)

2-cyano-N-(4-(1-methyl-1H-benzo[d]imidazol-2-yl)-3-( 
methylthio)-1-phenyl-1 $H$-Pyrazol-5-yl)acetamide (4) unde rwent Gewald thiophene synthes is via its reaction with cyanomethylene derivatives $(\mathbf{1 5 a}, \mathbf{b})$ and elemental sulfur in refluxing dioxane containing triethylamine as basic catalyst afforded the 3,5-diamino thiophene derivatives $(\mathbf{1 8 a}, \mathbf{b})$, respectively. Formation of (18a,b) proceed via non-isolable intermediates (16) and (17). The analytical and spectral data of compounds $(\mathbf{1 8 a}, \mathbf{b})$ are consistent with the proposed structures. The IR spectrum of (18a) displayed absorption bands at $3316,3218 \mathrm{~cm}^{-1}$ and $2198 \mathrm{~cm}^{-1}$ for the $\mathrm{NH}_{2}$ and cyano function and carbonyl absorption band at $1662 \mathrm{~cm}^{-1}$. Its ${ }^{1} \mathrm{H}$ NMR spectrum two singlet signals at $\delta 4.42 \mathrm{ppm}$ and $\delta$
$5.56 \mathrm{ppm}$ for $\mathrm{NH}_{2}$ protons and singlet signal at $\delta 10.21 \mathrm{ppm}$ assignable to the $\mathrm{NH}$ group. Its mass spectrum showed a molecular ion peak at $\mathrm{m} / \mathrm{z}=500$ corresponding to a mo lecular formula $\left(\mathrm{C}_{24} \mathrm{H}_{20} \mathrm{~N}_{8} \mathrm{OS}_{2}\right)$.

On the other hand reaction of (4) with phenyl isothiocayanate and elemental sulfur afforded the 4-a mino-2,3-dihydro-N-(4-(1-methyl-1 $H$-ben zo[d]imidazol2-yl)-3-(me-thylthio)-1-phenyl-1H-pyrazo 1-5-y l)-3-phenyl2-thio xothiazole-5-carboxamide (20) which proceed via intermediacy (16) and (19). The analytical and spectral data of compounds (18a,b) and (20) are consistent with the proposed structures (scheme 4).

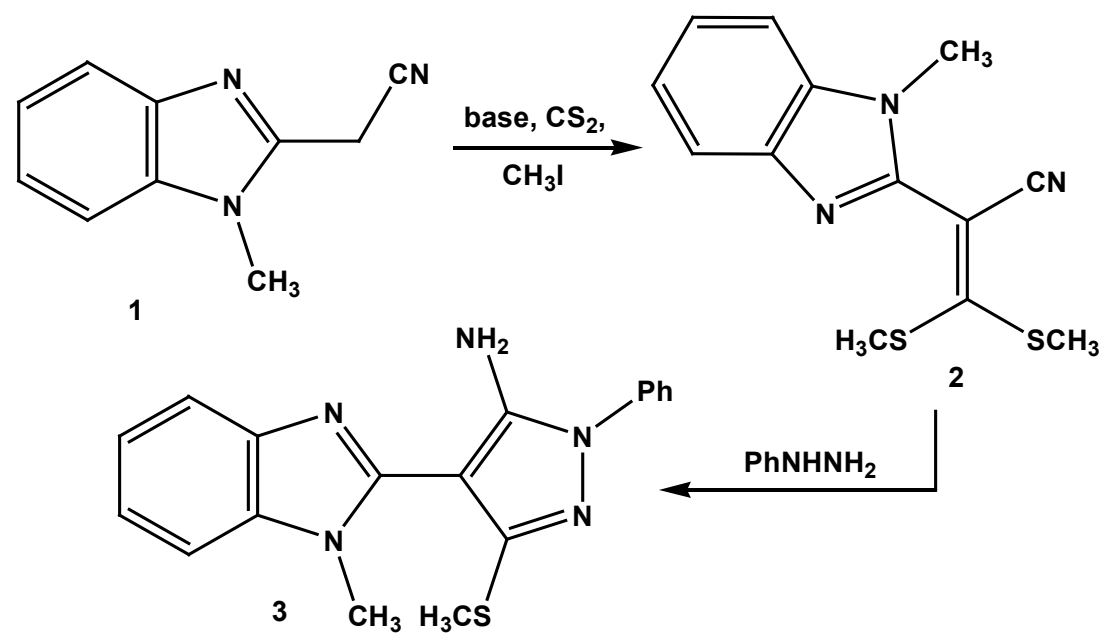

Scheme 1. Synthesis of 5-aminopyrazole derivative 3

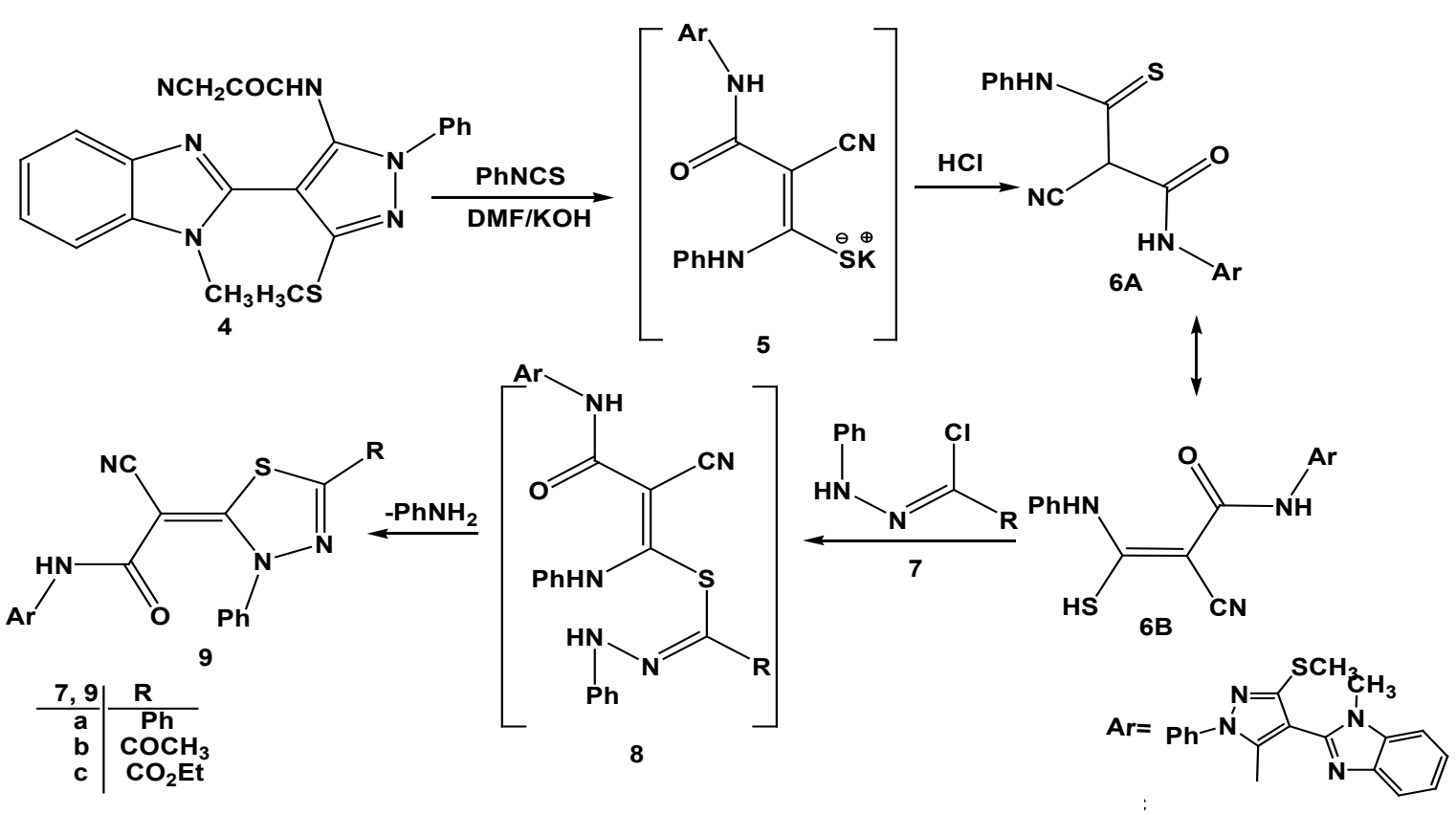

Scheme 2. Synthesis of 1,3,4-thiadiazole dericatives 9a-c 
5

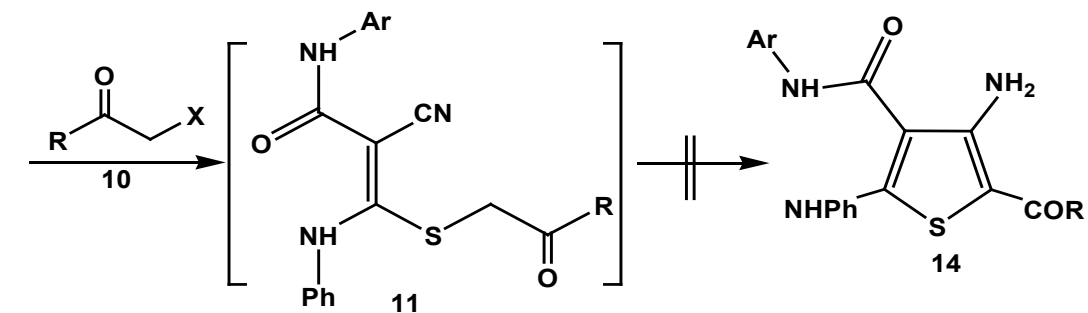<smiles></smiles>

12

\begin{tabular}{l|ll}
10,13 & $\mathrm{X}$ & $\mathrm{R}$ \\
\hline $\mathrm{a}$ & $\mathrm{Br}$ & $\mathrm{OEt}$ \\
$\mathrm{Br}$ & $\mathrm{CH}$ & \\
$\mathrm{C}$ & $\mathrm{Br}$ &
\end{tabular}

Scheme 3. Reactions of halocarbonyl compounds $10 \mathrm{a}-\mathrm{c}$ with enaminonitriles $11 \mathrm{a}-\mathrm{c}$

<smiles>[X]c1c(N)sc(C(=O)NCCCCCC)c1N</smiles>

18



Scheme 4. Synthesis of polysubst it uted thiophene $18 \mathrm{a}, \mathrm{b}$ and thiazole 20 derivatives 


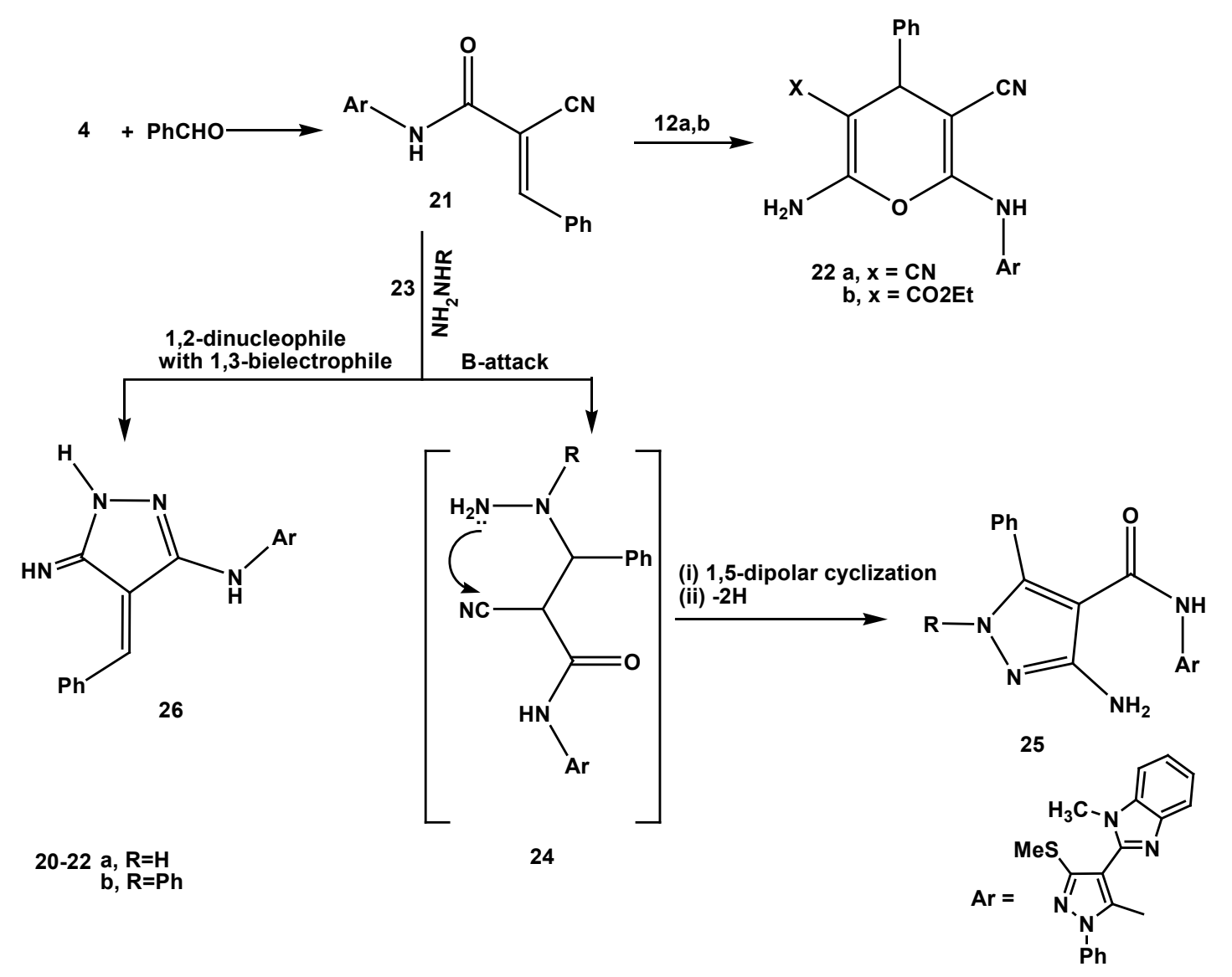

Scheme 5. Synthesis of pyrane 22a,b pyrazole derivatives 25 a, band 26

The reaction of2-cyano-N-(4-(1-methyl-1H-benzo[d] imidazo 1-2-yl)-3-(methylthio)-1-phenyl-1H-pyrazol-5-yl)ac etamide (4) with benzaldehyde yielded the phenyl-methylid ene derivative (21). The latter showed interesting reactivity towards cyanomethylene reagent $\mathbf{( 1 5 a , b )}$ afforded the2-(4-(1-methyl-1H-ben zo[d] imidazol- 2-yl)-3-(methylthi o)-1-phenyl-1 H-pyra zol-5-yla mino)-6-a mino-4-phenyl-4Hpyran-3,5-dicarbonitrile (22 a) and ethyl 6-(4-(1-methyl-1 Hbenzo[d]imidazol-2-yl)-3-(methyl-thio)-1-phenyl-1 $H$-pyraz ol-5-ylamino)-2-a mino-5-cyano-4-phenyl-4H-pyran-3-carb oxy late (22 b), respectively.

Also, when compound (21) reacted with hydrazine derivatives (23a, b) afforded the 3-amino-N-(4-(1- methyl$1 H$-benzo[d] imidazo l-2-yl)-3-(methylthio)-1-phenyl- $1 H$-pyr azol-5-yl)-1-phenyl-1H-pyrazo 1-5-yl)-5-phenyl- $1 H$-pyrazol e-4-carboxamide (25a) and 3-amino-N-(4-(1-methy $1-1 H$ benzo[d]imidazol-2-yl)-3-(methylthio)-1-phenyl-1H-pyrazo 1-5-yl)-1-phenyl-1 H-pyrazol-5-yl)-1,5-diphenyl-1H-pyrazol e-4-carboxa-mide $\mathbf{( 2 5} \mathbf{b})$ as the major products. The formation of the pyrazole derivatives $(\mathbf{2 5} \mathbf{a}, \mathbf{b})$ proceed via $\beta$-attack on the $\mathrm{C}=\mathrm{C}$ mo iety in (21) to give the non-isolable intermediate (24) which underwent 1,5-intramolecular dipolar cyclization and concomitant aromatization. Minor product (26) was obtained in the mother liquor which proceed via the condensation with the carbonyl followed by addition on the cyano function afforded the pyrazole derivatives (26) (scheme 5). The analytical and spectral data of compounds (22a, b), (25a, b) and (26) are consistent with the proposed structures.

\section{Experimental}

All organic solvents were purchased from commercial sources and used as received or dried using standard procedures, unless otherwise stated. All chemical were purchased from Aldrich or Across and used without purification. Melting points were measured on a Gallen kamp apparatus and are uncorrected. IR spectra were recorded on Shimadzu FT-IR 8101 PC infrared spectrophotometer. The ${ }^{1} \mathrm{H}$ NMR and ${ }^{13} \mathrm{C}$ NMR spectra were determined in DMSO-d6 at $300 \mathrm{MHz}$ on a Varian Mercury VX $300 \mathrm{NMR}$ spectrometer using TMS as an internal standard. Mass spectra were measured on a GCMS-QP1000 EX spectrometer at $70 \mathrm{Ev}$. Elemental analys es were carried out at the Microanalytical Center of Cairo University. (3,5-Dimethy-1H-pyrazol-1-yl)-3-oxopropanenitrile was prepared according to the reported literature[60], Hydrazonyl halides (7a) and (7 b,c) were prepared according to the reported literature[61] and[62,63], respectively. 4-(1-methyl-1H-benzo[d] imida zol-2-yl)-3-(methylthio)-1-P henyl-1H-pyrazo 1-5-amine (3) was prepared according to the reported literature[64].

2).2-Cyan o-N-(4-(1-me thyl-1 H-benzo[d]i mi dazol-2 -yl) -3-(meth ylthi o)-1 -phe nyl-1 H-pyr azol-5-yl)acetami de (4). 
166 Mohamed A. Khalil et al:: Reactivity of 2-Cyano-N-(4-(1-Methyl-1H-benzo[d]imidazol-2-yl)-3-(Methylthio)-1-Pheny l-1HPyrazol-5-yl)Acetamide: A Facile Synthesis of Pyrazole, Thiazole, 1,3,4-Thiadiazole and Poly substituted Thiophene Derivatives

A solution of4-(1-methyl-1H-benzo[d]imidazol-2-yl)-3(methylthio)-1-phenyl-1H-pyrazol-5-amine $(0.01 \mathrm{~mol})$ in dry toluene $(30 \mathrm{ml})$ was added to solution of with (3,5-dimethy -1H-pyrazol-1-yl)-3-oxopropanenitrile (0.01 mol) in dry toluene $(30 \mathrm{ml})$. The reaction mixture was refluxed for about $1 \mathrm{~h}$. After evaporation of the solvent, the solid product was collected by filtration and recrystallised fro $m$ dry DMF to afford (83\%, y ield) of (4), mp 293-295 C; IR $v_{\max } / \mathrm{cm}^{-1}(\mathrm{KBr}) 3215,3172(\mathrm{NH}), 2218(\mathrm{CN}), 1658(\mathrm{CO})$, $1630(\mathrm{C}=\mathrm{N}) ;{ }^{1} \mathrm{H}$ NMR $\left(\mathrm{DMSO}_{6}\right) \delta 2.68\left(\mathrm{~s}, 3 \mathrm{H}, \mathrm{SCH}_{3}\right)$, 3.91(s, 3H, $\left.\mathrm{NCH}_{3}\right), 4.12\left(\mathrm{~s}, 2 \mathrm{H}, \mathrm{CH}_{2}\right), 7.51-8.01(\mathrm{~m}, 9 \mathrm{H}$, Ar-H), 10.65 (s, 1H, NH); m/z $402\left(\mathrm{M}^{+}, 13.75\right)$. Anal. Calcd. For $\mathrm{C}_{21} \mathrm{H}_{18} \mathrm{~N}_{6} \mathrm{OS}$ (402.47): C, 62.67; H, 4.51; N, 20.88; S, 7.97\%. Found:. C, 62.63; H, 4.49; N, 20.78; S, 7.94\%.

3).2-Cyan o-3-mercapto-N-(4-(1-methyl-1 H-benzo[d]i mi dazol -2-yl )-3-me th ylthi o-1 - phen yl-1 H-pyraz ol-5-yl)-3 phenylamino acrylami de (6).

To stirred solution of potassium hydroxide $(0.01 \mathrm{~mol})$ in dimethylformamide (20ml), 2-cyano-N-(4-(1-methyl-1Hbenzo[d]imidazol-2-yl)-3-(methylthio)-1-phenyl-1H-pyrazo 1-5-yl)acetamide (4) (0.01 mol) was added. After stirring for $30 \mathrm{~min}$., phenyl isothiocyanate $(0.01 \mathrm{~mol})$ was added to the resulting mixture. Stirring was continued overnight then poured onto crushed ice containing hydrochloric acid. The solid product so-formed was collected by filtration, washed with water, dried and finally recrystallized from ethanol/dimethylformamide to afford (72\%, yield) of (6), mp 239-241 C; IR $v_{\max } / \mathrm{cm}^{-1}(\mathrm{KBr}) 3362,3215(\mathrm{NH}), 2206$ $(\mathrm{CN}), 1653(\mathrm{CO}), 1635(\mathrm{C}=\mathrm{N}) ;{ }^{1} \mathrm{H}$ NMR $\left(\mathrm{DMSO}_{6}\right) \delta 2.68$ (s, $\left.3 \mathrm{H}, \mathrm{SCH}_{3}\right), 3.89$ (s, 3H, $\left.\mathrm{NCH}_{3}\right), 7.35-7.98(\mathrm{~m}, 14 \mathrm{H}, \mathrm{Ar}-\mathrm{H})$, 9.85 (s, 1H, NH), 10.65 (s, 1H, NH), 13.84 (s, 1H, SH); m/z $538\left(\mathrm{M}^{+}+1,26.12\right)$. Anal. Calcd. For $\mathrm{C}_{28} \mathrm{H}_{23} \mathrm{~N}_{7} \mathrm{OS}_{2}$ (537.66): C, 62.55; H, 4.31; N, 18.24; S, 11.93\%. Found:. C, 62.53; H, 4.28 ; N, 18.19; S, $11.99 \%$.

\subsection{Gener al Procedure for the Reaction of}

2-Cyano-3-mercapto-N-(4-(1-me thyl-1 H-benzo[d]i m idazol-2-yl)-3-meth ylthi o-1-phen yl-1 H-pyrazol-5-yl) -3-phenyl amino acryl ami de (6) wi th Hydrazonoyl Chlorides (7 a-c)

To a solution of (6) $(1 \mathrm{mmol})$ in ethanol $(20 \mathrm{ml})$ and the appropriate hydrazonoyl chlorides (7a-c) (1 mmol of each), triethylamine $(0.5 \mathrm{ml})$ was added. The reaction mixture was refluxed for $4 \mathrm{~h}$., then allowed to cool. The formed solid was collected by filtration, washed with ethanol and recrystallized from the suitable solvent to afford the corres ponding 1, 3, 4-th iadia zole derivatives (9a-c).

1).2-Cyano-N-(4-(1-methyl-1H-benzo[d]imidazol-2-yl)-3 -(methylthio)-1-p henyl-1 H-pyrazol-5-yl)-2-(3,5-diphenyl-1 ,3,4-thiadiazol-2(3H)ylidene)acetamide (9a).

This compound was obtained as orange-red powder (DMF/EtOH 1:2), (53\%, y ield), mp 208-210 C; IR $v_{\max } /$ $\mathrm{cm}^{-1}(\mathrm{KBr}) 3237(\mathrm{NH}), 2208(\mathrm{CN}), 1662(\mathrm{CO}), 1632(\mathrm{C}=\mathrm{N})$; ${ }^{1} \mathrm{H}$ NMR $\left(\mathrm{DMSO}_{-} \mathrm{d}_{6}\right) \delta 2.66\left(\mathrm{~s}, 3 \mathrm{H}, \mathrm{SCH}_{3}\right), 3.86(\mathrm{~s}, 3 \mathrm{H}$, $\mathrm{NCH}_{3}$ ), 7.37-8.12 (m, 19H, Ar-H), 9.98 (s, 1H, NH); m/z 638 $\left(\mathrm{M}^{+}\right.$, 28.34). Anal. Calcd. For $\mathrm{C}_{35} \mathrm{H}_{26} \mathrm{~N}_{8} \mathrm{OS}_{2}$ (638.76): $\mathrm{C}$,
65.81; H, 4.10; N, 17.54; S, 10.04\%. Found:. C, 65.78; H, 4. $10 ; \mathrm{N}, 17.52 ; \mathrm{S}, 9.99 \%$.

2).2-(5-Acetyl-3-phenyl-1,3,4-thiadiazol-2(3H)-ylidene)2-cyano-N-(4-(1-methyl-1H-benzo[d]imidazol-2-yl)-3-(met hylthio)-1-phenyl-1H-pyrazol-5-yl)acetamide (9b).

This compound was obtained as brownish-red crystals (DMF/EtOH 1:20), (55\%, yield), mp 281-283 C; IR $v_{\max } /$ $\mathrm{cm}^{-1}(\mathrm{KBr}) 3232(\mathrm{NH}), 2198(\mathrm{CN}), 1650 ; 1683(\mathrm{CO}), 1630$ $(\mathrm{C}=\mathrm{N}) ;{ }^{1} \mathrm{H}$ NMR $\left(\mathrm{DMSO}_{6}\right) \delta 2.69\left(\mathrm{~s}, 3 \mathrm{H}, \mathrm{SCH}_{3}\right), 2.72(\mathrm{~s}$, $\left.3 \mathrm{H}, \mathrm{COCH}_{3}\right), 3.90$ (s, 3H, NCH 3$), 7.34-8.07$ (m, 14H, Ar-H), $10.15(\mathrm{~s}, 1 \mathrm{H}, \mathrm{NH}) ; \mathrm{m} / \mathrm{z} 604\left(\mathrm{M}^{+}, 21.45\right)$. Anal. Calcd. For $\mathrm{C}_{31} \mathrm{H}_{24} \mathrm{~N}_{8} \mathrm{O}_{2} \mathrm{~S}_{2}$ (604.70): C, 61.57; H, 4.00; N, 18.53; S, 10.61\%. Found:. C, 61.55; H, 4.10; N, 18.51; S, 10.58\%.

3).Ethyl 5-((4-(1-methyl-1H-benzo[d]imidazol-2-yl)-3 -(methylthio)-1-phenyl-1H-pyr-azol-5-ylcarbamoyl)(cyano )methylene)-4,5-dihydro-4-phenyl-1,3,4-thiadiazol-2-carbo xylate (9c).

This compound was obtained as pal brown crystalsr (DMF/EtOH), (42\%, yield), mp 241-243 C; IR $v_{\max } / \mathrm{cm}^{-1}$ (KBr) $3234(\mathrm{NH}), 2203(\mathrm{CN}), 1732$ (CO), 1655 (CO), 1631 $(\mathrm{C}=\mathrm{N}) ;{ }^{1} \mathrm{H}$ NMR $\left(\mathrm{DMSO}-\mathrm{d}_{6}\right) \delta 1.45\left(\mathrm{t}, 3 \mathrm{H}, \mathrm{J}=7.2 \mathrm{~Hz}, \mathrm{CH}_{3}\right.$ ester), 2.69 (s, 3H, $\left.\mathrm{SCH}_{3}\right), 3.92\left(\mathrm{~s}, 3 \mathrm{H}, \mathrm{NCH}_{3}\right), 4.52$ ( q, $2 \mathrm{H}, \mathrm{J}$ $=7.2 \mathrm{~Hz}, \mathrm{CH}_{2}$ ester), 7.31-8.11 (m, 14H, Ar-H), $10.18(\mathrm{~s}, 1 \mathrm{H}$, $\mathrm{NH}) ; \mathrm{m} / \mathrm{z} 635\left(\mathrm{M}^{+}, 28.34\right)$. Anal. Calcd. For $\mathrm{C}_{32} \mathrm{H}_{26} \mathrm{~N}_{8} \mathrm{O}_{3} \mathrm{~S}_{2}$ (634.73): C, 60.55; H, 4.13; N, 17.65; S, 10.10\%. Found:. C, $60.53 ; \mathrm{H}, 4.11 ; \mathrm{N}, 17.61 ; \mathrm{S}, 10.10 \%$.

\subsection{Gener al Procedure for the synthesis of 2-Cyano} -N-(4-(1-methyl-1 H-be nzo[d]-i mi dazol-2-yl)-3-(me th ylthi o)-1 -phe nyl-1 H-pyr azol -5-yl)-2-(4 -oxo-3-phen yl thiazol-i din-2-yli dene)acetami de (12), 2-Cyano-N-(4 -(1-methyl-1 H-benzo[d]i mi daz ol-2-yl)-3-(me thylthi o )-1-phen yl-1 H-pyr az ol-5-yl)-2-(4-meth yl-3-phen ylthi azol-2 $(3 \mathrm{H})$-yli dene)-acetami de $(13 \mathrm{~b})$ and 2-(4-(be nzo [d]thiaz ol-2-yl)-3-phen ylthiaz ol-2 (3 H)-yli de ne-2-cyan o-N-(4-(1-me th yl-1 H-benzo[d]i mi dazol2-yl)-3-(methyl thio)-1-phe nyl-1 H-pyr azol-5-yl) acetami de (13c)

To stirred solution of potassium hydroxide $(10 \mathrm{mmol})$ in dimethylformamide $\quad(20 \mathrm{ml}), \quad$ 2-cyano-N-(4-(1-methy 1- $1 H$-benzo[d] imidazol-2-yl)-3-(methylthio)-1-phenyl- $1 H$-p yrazol-5-yl)acetamide (4) (10 mmol) was added. After stirring for $30 \mathrm{~min}$., phenyl is othiocyanate $(10 \mathrm{mmol})$ was added to the resulting mixture. Stirring was continued 6h., and then the appropriate $\alpha$-halocarbonyl compounds (10a-c) (10 mmol of each) was added. Stirring continued for additional overnight. Then, the reaction mixture was poured onto crushed ice water. The solid product that formed was filtered off, dried and recrystallized fro $m$ the suitable solvent to afford the corresponding thiazole derivatives (12) and $(\mathbf{1 3 b}, \mathbf{c})$, respectively.

1).2-Cyano-N-(4-(1-methyl-1H-benzo[d]-imidazol-2-yl)3-(methylthio)-1-phenyl-1H-pyrazol-5-yl)-2-(4-oxo-3-phen ylthiazolidin-2-ylidene)acetamide (12).

This compound was obtained as yellow powder (DMF), (65\%, yield), mp 266-268 C; IR v $v_{\max } / \mathrm{c} \mathrm{m}^{-1}$ (KBr) $3347(\mathrm{NH})$, 
$2223(\mathrm{CN}), 1685(\mathrm{CO}), 1648(\mathrm{CO}), 1630(\mathrm{C}=\mathrm{N}) ;{ }^{1} \mathrm{H}$ NMR $\left(\mathrm{DMSO}_{\mathrm{d}}\right) \delta 2.69\left(\mathrm{~s}, 3 \mathrm{H}, \mathrm{SCH}_{3}\right), 3.73\left(\mathrm{~s}, 2 \mathrm{H}, \mathrm{CH}_{2}\right), 3.89(\mathrm{~s}$, $\left.3 \mathrm{H}, \mathrm{NCH}_{3}\right), 7.38-7.97(\mathrm{~m}, 14 \mathrm{H}, \mathrm{Ar}-\mathrm{H}), 10.31(\mathrm{~s}, 1 \mathrm{H}, \mathrm{NH})$; $\mathrm{m} / \mathrm{z} 577\left(\mathrm{M}^{+}\right.$, 19.27). Anal. Calcd. For $\mathrm{C}_{30} \mathrm{H}_{23} \mathrm{~N}_{7} \mathrm{O}_{2} \mathrm{~S}_{2}$ (577.68): C, 62.37; H, 4.01; N, 16.97; S, 11.16\%. Found:. C, $62.34 ; \mathrm{H}, 3.99 ; \mathrm{N}, 16.87 ; \mathrm{S}, 11.10 \%$

2).2-Cyano-N-(4-(1-methyl-1H-benzo[d]imidazol-2-yl)-3 -(methylthio)-1-phenyl-1 H-pyrazol-5-yl)-2-(4-methyl-3phenylthiazol-2(3H)-ylidene)acetamide (13b).

This compound was obtained as pale brown powder (DMF/Et OH), $\left(46 \%\right.$, yield), $\mathrm{mp}>300^{\circ} \mathrm{C}$; IR $v_{\max } / \mathrm{cm}^{-1}(\mathrm{KBr})$ $3218(\mathrm{NH}), 2204(\mathrm{CN}), 1663(\mathrm{CO}), 1630(\mathrm{C}=\mathrm{N}) ;{ }^{1} \mathrm{H}$ NMR $\left(\mathrm{DMSO}_{\mathrm{d}}\right) \delta 1.72\left(\mathrm{~s}, 3 \mathrm{H}, \mathrm{CH}_{3}\right), 2.70\left(\mathrm{~s}, 3 \mathrm{H}, \mathrm{SCH}_{3}\right), 3.89(\mathrm{~s}$, $\left.3 \mathrm{H}, \mathrm{NCH}_{3}\right), 6.93(\mathrm{~s}, 1 \mathrm{H},=\mathrm{CH}), 7.27-7.92(\mathrm{~m}, 14 \mathrm{H}, \mathrm{Ar}-\mathrm{H})$, 10.53 (s, 1H, NH); m/z $575\left(\mathrm{M}^{+}, 19.27\right)$. Anal. Calcd. For $\mathrm{C}_{31} \mathrm{H}_{25} \mathrm{~N}_{7} \mathrm{OS}_{2}$ (575.71): C, 64.67; H, 4.38; N, 17.03; S, $11.14 \%$. Found: C, 62.61; H, 4.36; N, 17.10; S, 11.11\%.

3).2-(4-(Benzo[d]thiazol-2-yl)-3-phenylthiazol-2(3H)-yli dene-2-cyano-N-(4-(1-methyl-1H-benzo[d]imidazol-2-yl)-3 -(methylthio)-1-phenyl-1 H-pyrazol-5-yl)acetamide (13c).

This compound was obtained as dark brown crystals (DMF), (61\%, yield), $\mathrm{mp}>300^{\circ} \mathrm{C}$; IR $v_{\max } / \mathrm{cm}^{-1}(\mathrm{KBr}) 3243$ $(\mathrm{NH}), 2214(\mathrm{CN}), 1665(\mathrm{CO}), 1632(\mathrm{C}=\mathrm{N}) ;{ }^{1} \mathrm{H}$ NMR $\left(\mathrm{DMSO}_{\mathrm{d}} \mathrm{d}_{6}\right) \delta 2.70\left(\mathrm{~s}, 3 \mathrm{H}, \mathrm{SCH}_{3}\right), 3.88\left(\mathrm{~s}, 3 \mathrm{H}, \mathrm{NCH}_{3}\right), 6.89(\mathrm{~s}$, $1 \mathrm{H},=\mathrm{CH}), 7.42-8.21(\mathrm{~m}, 18 \mathrm{H}, \mathrm{Ar}-\mathrm{H}), 10.49(\mathrm{~s}, 1 \mathrm{H}, \mathrm{NH}) ; \mathrm{m} / \mathrm{z}$ $694\left(\mathrm{M}^{+}, 19.27\right)$. Anal. Calcd. For $\mathrm{C}_{37} \mathrm{H}_{26} \mathrm{~N}_{8} \mathrm{OS}_{3}$ (694.85): $\mathrm{C}$, 63.96; H, 3.77; N, 16.13; S, 13.84\%. Found:. C, 63.97; H, 3.78 ; N, 16.11; S, $13.76 \%$.

\subsection{General Procedure for the synthesis of}

3,5-Diamin o-4-cyano-N-(4-(1 -meth yl-1 H-benzo[d]i m idazol-2-yl)-3-(me th ylthio)-1-phenyl-1 H-pyr azol-5-y 1)thiophene-2-carbo-xamide(18a) and Ethyl

5-(4-(1-me thyl-1 H-benzo[d]-imi dazol-2-yl)-3-(methy Ithio)-1-phenyl-1 H-pyr azol-5-ylcar bamoyl)-2,4-di a minothiophene-3-car boxylate (18b)

To a solution of compound (4) $(0.01 \mathrm{~mol})$ in 1,4-dioxane $(30 \mathrm{ml})$ containing triethyla mine $(1 \mathrm{ml})$, either malononitrile $(0.01 \mathrm{~mol})$ or ethyl cyanoacetate $(0.01 \mathrm{~mol})$ was added followed by the addition of an equimolar amount of elemental sulfur $(0.01 \mathrm{~mol})$. The reaction mixture was heated under reflux for $5 \mathrm{~h}$., then cooled and neutralized by pouring onto ice/water mixture containing few drops of hydrochloric acid. The solid product formed in each case was collected by filtration and crystallized fro $m$ dimethylformamide.

1).3,5-Diamino-4-cyano- $N$-(4-(1-methyl-1H-benzo[d]im idazol-2-yl)-3-(methylthio)-1-phenyl-1H-pyrazol-5-yl)thiop hene-2-carboxamide (18a).

Dark brown crystals, $\left(60 \%\right.$, yield), mp $>300^{\circ} \mathrm{C}$; IR $v_{\max } /$ $\mathrm{cm}^{-1}(\mathrm{KBr})$ 3316-3218 (NH and $\left.\mathrm{NH}_{2}\right), 2198(\mathrm{CN}), 1662$ (CO), $1625(\mathrm{C}=\mathrm{N}) ;{ }^{1} \mathrm{H}$ NMR $\left(\mathrm{DMSO}_{6}\right) \delta 2.71(\mathrm{~s}, 3 \mathrm{H}$, $\left.\mathrm{SCH}_{3}\right), 3.91\left(\mathrm{~s}, 3 \mathrm{H}, \mathrm{NCH}_{3}\right), 4.42\left(2 \mathrm{~s}, 2 \mathrm{H}, \mathrm{NH}_{2}\right), 4.56(2 \mathrm{~s}, 2 \mathrm{H}$, $\mathrm{NH}_{2}$ ), 7.22-7.98 (m, 9H, Ar-H), $10.21(\mathrm{~s}, 1 \mathrm{H}, \mathrm{NH}) ; \mathrm{m} / \mathrm{z} 500$ $\left(\mathrm{M}^{+}, 13.34\right)$. Anal. Calcd. For $\mathrm{C}_{24} \mathrm{H}_{20} \mathrm{~N}_{8} \mathrm{OS}_{2}$ (500.6): C, 57.58; H, 4.03; N, 22.38; S, 12.81\%. Found: C, 57.56; H, 4.05; N, $22.36 ; \mathrm{S}, 12.79 \%$.

2).Ethyl 5-(4-(1-methyl-1H-benzo[d]imidazol-2-yl)-3- (methylthio)-1-phenyl-1 H-pyr-azol-5-ylcarbamoyl)-2,4-dia minothiophene-3-carboxylate (18b).

Brown crystals, $\left(56 \%\right.$, yield), $\mathrm{mp}>300^{\circ} \mathrm{C}$; IR $v_{\max } / \mathrm{cm}^{-1}$ $(\mathrm{KBr})$ 3436-3385( $\left.\mathrm{NH}_{2}\right), 3256(\mathrm{NH}), 2205(\mathrm{CN}), 1696,1650$ (2CO), $1625(\mathrm{C}=\mathrm{N}) ;{ }^{1} \mathrm{H}$ NMR $\left(\mathrm{DMSO}-\mathrm{d}_{6}\right) \delta 1.12(\mathrm{t}, \mathrm{J}=7.00$ $\left.\mathrm{Hz}, 3 \mathrm{H}, \mathrm{CH}_{3}\right), 2.70\left(\mathrm{~s}, 3 \mathrm{H}, \mathrm{SCH}_{3}\right), 3.49$ (q, J $=7.00 \mathrm{~Hz}, 2 \mathrm{H}$, $\mathrm{CH}_{2}$ ), 3.90(s, 3H, $\mathrm{NCH}_{3}$ ), 3.98 (s, $\left.2 \mathrm{H}, \mathrm{NH}_{2}\right), 4.15$ (s, $2 \mathrm{H}$, $\mathrm{NH}_{2}$ ), 7.23-7.96 (m, 9H, Ar-H), 10.23 (s, 1H, NH); m/ z 547 $\left(\mathrm{M}^{+}, 12.11\right)$. Anal. Caled. For $\mathrm{C}_{26} \mathrm{H}_{25} \mathrm{~N}_{7} \mathrm{O}_{3} \mathrm{~S}_{2}$ (547.65): $\mathrm{C}$, $57.02 ; \mathrm{H}, 4.60 ; \mathrm{N}, 17.90 ; \mathrm{S}, 11.71 \%$. Found: C, 57.10; H, $4.57 ; \mathrm{N}, 17.88 ; \mathrm{S}, 11.75 \%$.

3).4-Amino-2,3-dihydro-N-(4-(1-methyl-1 H-benzo [d]imidazol-2-yl)-3-(methylthio)-1-phenyl-1 H-pyrazol-5-yl )-3-phenyl-2-thioxothiazole-5-carboxamide (20)

To a solution of compound (4) $(0.01 \mathrm{~mol})$ in absolute ethanol $(30 \mathrm{ml})$ containing triethylamine $(1 \mathrm{ml})$ and elemental sulfur $(0.01 \mathrm{~mol})$ was added followed by the addition of an equimolar amount of phenyl isothiocyanate $(0.01 \mathrm{~mol})$. The reaction mixture was heated under reflux for $1 \mathrm{~h}$., at $75^{\circ} \mathrm{C}$ with continuous stirring and then cooled and neutralized by pouring onto ice/water mixture containing few drops of hydrochloric acid. The solid product formed was collected by filtration and crystallized from dimethylformamide and ethanol.

White powder, (52\%, y ield), mp 295-297 C; IR $v_{\max } / \mathrm{cm}^{-1}$ $(\mathrm{KBr})$ 3421-3382 ( $\left.\mathrm{NH}_{2}\right), 3250(\mathrm{NH}), 2208(\mathrm{CN}), 1650(\mathrm{CO})$, $1628(\mathrm{C}=\mathrm{N}) ;{ }^{1} \mathrm{H}$ NMR $\left(\right.$ DMSO-d $\left._{6}\right) \delta 2.70\left(\mathrm{~s}, 3 \mathrm{H}, \mathrm{SCH}_{3}\right)$, $5.11\left(\mathrm{~s}, 2 \mathrm{H}, \mathrm{NH}_{2}\right), 3.90\left(\mathrm{~s}, 3 \mathrm{H}, \mathrm{NCH}_{3}\right), 7.21-7.92(\mathrm{~m}, 14 \mathrm{H}$, Ar-H), 10.19(s, 1H, NH); m/ z $569\left(\mathrm{M}^{+}, 53.11\right)$. Anal. Calcd. For $\mathrm{C}_{28} \mathrm{H}_{23} \mathrm{~N}_{7} \mathrm{OS}_{3}$ (569.72): C, 59.03; H, 4.07; N, 17.21; $\mathrm{S}$, $16.88 \%$. Found: C, 59.10; H, 4.10; N, 17.18; S, 16.79\%.

4).2-Cyano-N-(4-(1-methyl-1H-benzo[d]imidazol-2-yl)-3 -(methylthio)-1-phenyl-1 H-pyrazol-5-yl)-3-phenylacrylami de (21).

To a solution of compound (4) $(0.01 \mathrm{~mol})$ in 1,4-dioxane $(30 \mathrm{ml})$ containing piperidine $(0.5 \mathrm{ml})$, benzaldehyde $(0.01$ mol) was added. The reaction mixture was heated under reflux for $3 \mathrm{~h}$., then left to cool. The solid product so formed was collected by filtration and crystallized from ethanol.

Pale yellow crystals, (46\%, yield), mp $215-217^{\circ} \mathrm{C}$; IR $v_{\max }$ $/ \mathrm{cm}^{-1}(\mathrm{KBr}) 3250(\mathrm{NH}), 2221(\mathrm{CN}), 1679(\mathrm{CO}), 1637(\mathrm{C}=\mathrm{N})$; ${ }^{1} \mathrm{H}$ NMR $\left(\mathrm{DMSO}_{-} \mathrm{d}_{6}\right) \delta 2.71\left(\mathrm{~s}, 3 \mathrm{H}, \mathrm{SCH}_{3}\right), 3.90(\mathrm{~s}, 3 \mathrm{H}$, $\left.\mathrm{NCH}_{3}\right), 6.25(\mathrm{~s}, 1 \mathrm{H}, \mathrm{CH}=\mathrm{C}), 7.21-7.91(\mathrm{~m}, 14 \mathrm{H}, \mathrm{Ar}-\mathrm{H})$, $10.28(\mathrm{~s}, 1 \mathrm{H}, \mathrm{NH}) ; \mathrm{m} / \mathrm{z} 490\left(\mathrm{M}^{+}, 27.32\right)$. Anal. Calcd. For $\mathrm{C}_{28} \mathrm{H}_{22} \mathrm{~N}_{6} \mathrm{OS}(490.58)$ : C, 68.55; H, 4.52; N, 17.13; S, 6.54\%. Found: C, 68.53; H, 4.49; N, 17.11; S, 6.56\%.

\subsection{General Procedure for the synthesis of}

2-(4-(1-Methyl-1 H-be nzo [d]imi daz ol-2-yl)-3-(methyl thio)-1-phenyl-1 H-pyrazol-5-ylami no)-6-amino-4-ph enyl-4 H-pyran-3,5-dic-arbonitrile (22a) and Ethyl 6-(4-(1-me thyl-1 H-benzo[d]i mi dazol-2 -yl)-3-(methyl -lthio)-1-phe nyl-1 H-pyrazol-5-ylami no)-2-amino-5cyano-4-phenyl-4 H-pyr an-3-car boxylate (22 b)

To a solution of compound (21) $(0.01 \mathrm{~mol})$ in 1,4-dio xane $(30 \mathrm{ml})$ containing triethylamine $(0.5 \mathrm{ml})$, either malononitrile $(0.01 \mathrm{~mol})$ or ethyl cyanoacetate $(0.01 \mathrm{~mol})$ 
was added. The reaction mixture was heated in each case under reflux for $6 \mathrm{~h}$., then the excess solvent was evaporated under reduced pressure. The solid product formed in each case was collected by filtration and crystallized from dimethylforma mide/ethanol (2:1).

1).2-(4-(1-Methyl-1 H-benzo[d]imidazol-2-yl)-3-(methylt hio)-1-phenyl-1H-pyrazol-5-ylamino)-6-amino-4-phenyl-4 H-pyran-3,5-dicarbonitrile (22a).

White crystals, $\left(52 \%\right.$, yield), mp $267-269^{\circ} \mathrm{C}$; IR $v_{\max } / \mathrm{cm}^{-1}$ (KBr) $3345\left(\mathrm{NH}_{2}\right), 3255(\mathrm{NH}), 2222,2219(2 \mathrm{CN}), 1650$ (CO), $1635(\mathrm{C}=\mathrm{N}) ;{ }^{1} \mathrm{H}$ NMR $\left(\mathrm{DMSO}_{-} \mathrm{d}_{6}\right) \delta 2.70(\mathrm{~s}, 3 \mathrm{H}$, $\left.\mathrm{SCH}_{3}\right), 3.90$ (s, $\left.3 \mathrm{H}, \mathrm{NCH}_{3}\right), 4.76\left(\mathrm{~s}, 2 \mathrm{H}, \mathrm{NH}_{2}\right), 6.35(\mathrm{~s}, 1 \mathrm{H}$, pyran $\mathrm{H}-4), 7.21-7.84(\mathrm{~m}, 14 \mathrm{H}, \mathrm{Ar}-\mathrm{H}), 10.23(\mathrm{~s}, 1 \mathrm{H}, \mathrm{NH})$; $\mathrm{m} / \mathrm{z} 556\left(\mathrm{M}^{+}, 53.11\right)$. Anal. Calcd. For $\mathrm{C}_{31} \mathrm{H}_{24} \mathrm{~N}_{8} \mathrm{~S}(556.64)$ : C, 66.89; H, 4.35; N, 20.13; S, 5.76\%. Found: C, 66.86; H, $4.31 ; \mathrm{N}, 20.12 ; \mathrm{S}, 5.75 \%$.

2).Ethyl 6-(4-(1-methyl-1H-benzo[d]imidazol-2-yl)-3 -(methylthio)-1-p henyl-1H-pyra-zol-5-ylamino)-2-amino-5 -cyano-4-phenyl-4H-pyran-3-carboxylate (22b).

Pale yellow crystals, $\left(51 \%\right.$, yield), mp $201-203^{\circ} \mathrm{C}$; IR $v_{\max }$ $/ \mathrm{cm}^{-1}(\mathrm{KBr}) 3368\left(\mathrm{NH}_{2}\right), 3259(\mathrm{NH}), 2222(\mathrm{CN}), 1698,1652$ (2CO), $1629(\mathrm{C}=\mathrm{N}) ;{ }^{1} \mathrm{H}$ NMR $\left(\mathrm{DMSO}_{-} \mathrm{d}_{6}\right) \delta 1.33(\mathrm{t}, 3 \mathrm{H}, \mathrm{J}=$ $\left.6.78 \mathrm{~Hz}, \mathrm{CH}_{3}\right), 2.72\left(\mathrm{~s}, 3 \mathrm{H}, \mathrm{SCH}_{3}\right), 3.90\left(\mathrm{~s}, 3 \mathrm{H}, \mathrm{NCH}_{3}\right), 4.24$ (q, $\left.2 \mathrm{H}, \mathrm{J}=6.78 \mathrm{~Hz}, \mathrm{CH}_{2}\right), 4.83\left(\mathrm{~s}, 2 \mathrm{H}, \mathrm{NH}_{2}\right), 6.62(\mathrm{~s}, 1 \mathrm{H}$, pyran $\mathrm{H}-4), 7.31-7.81(\mathrm{~m}, 14 \mathrm{H}, \mathrm{Ar}-\mathrm{H}), 10.21(\mathrm{~s}, 1 \mathrm{H}, \mathrm{NH})$; $\mathrm{m} / \mathrm{z} 603\left(\mathrm{M}^{+}\right.$, 32.54). Anal. Calcd. For $\mathrm{C}_{33} \mathrm{H}_{29} \mathrm{~N}_{7} \mathrm{O}_{3} \mathrm{~S}$ (603.69): C, 66.65; H, 4.84; N, 16.24; S, 5.31\%. Found: C, $66.63 ; \mathrm{H}, 4.82 ; \mathrm{N}, 16.19 ; \mathrm{S}, 5.30 \%$.

\subsection{General Procedure for the synthesis of}

3-Amino-N-(4-(1-me th yl-1 H-benzo-[d]-i mi dazol-2-yl )-3-(me thylthio)-1-phen yl-1 H-pyr azol-5-yl)-5-phenyl -1 H-pyr azole-4-c ar boxami de (25a),

3-Amino-N-(4-(1-me thyl-1 H-benzo[d]i mi d-azol-2-yl) -3-(methyl-thio)-1-phenyl-1 H-pyrazol-5-yl)-1,5-diph enyl-1 $\mathrm{H}$-pyrazole-4-carboxami mi de $(25 \mathrm{~b})$ and N-(4-benzyli dene-4,5-dihydr 0-5-a min o-1 H-pyrazol3-yl)-4-(1-me th yl-1 H-benzo[d]i mi dazol-2-yl)-3-(met hylthio)-1-phenyl-1 H-pyrazol-5-amine (26)

To a solution of compound (21) $(0.01 \mathrm{~mol})$ in 1,4-dio xane $(25 \mathrm{ml})$ and dimethylformamide $(5 \mathrm{ml})$, either hydrazine hydrate $(0.01 \mathrm{~mol})$ or phenyl hydrazine $(0.01 \mathrm{~mol})$ was added. The reaction mixture in each case was heated under reflux for $6 \mathrm{~h}$. The solid products formed, were collected by filtration, and crystallized from dimethylformamide and 1,4-dio xane mixture. The mother liquor in case of hydrazine hydrate pouring onto ice/water mixture containing few drops of hydrochloric acid. The solid products formed, were collected by filtration, and crystallized from dimethylformamide.

1).3-Amino-N-(4-(1-methyl-1 H-be nzo[d]imi dazol-2-yl) -3-(methylthi o)-1 -phenyl-1 H-pyr azol-5 -yl)-5-phenyl-1 Hpyrazole-4-carboxami de (25a).

Pale brown crystals, $\left(51 \%\right.$, yield), $m p 241-243^{\circ} \mathrm{C}$; IR $v_{\max } /$ $\mathrm{cm}^{-1}(\mathrm{KBr}) 3442,3259\left(2 \mathrm{NH}\right.$ and $\left.\mathrm{NH}_{2}\right), 1645(\mathrm{CO}), 1629$ $(\mathrm{C}=\mathrm{N}) ;{ }^{1} \mathrm{H}$ NMR $\left(\mathrm{DMSO}^{-\mathrm{d}_{6}}\right) \delta 2.72\left(\mathrm{~s}, 3 \mathrm{H}, \mathrm{SCH}_{3}\right), 3.90(\mathrm{~s}$, $\left.3 \mathrm{H}, \mathrm{NCH}_{3}\right), 4.23\left(\mathrm{~s}, 2 \mathrm{H}, \mathrm{NH}_{2}\right), 7.31-7.83(\mathrm{~m}, 14 \mathrm{H}, \mathrm{Ar}-\mathrm{H})$, $10.21(\mathrm{~s}, 1 \mathrm{H}, \mathrm{NH}) ; 13.10(\mathrm{~s}, 1 \mathrm{H}, \mathrm{NH}) ; \mathrm{m} / \mathrm{z} 520\left(\mathrm{M}^{+}, 16.56\right)$. Anal. Calcd. For $\mathrm{C}_{28} \mathrm{H}_{24} \mathrm{~N}_{8} \mathrm{OS}(520.61)$ : C, 64.60; H, 4.65; N, 21.52 ; S, 6.16\%. Found: C, 64.58; H, 4.61; N, 21.48; S, $6.13 \%$.

2).3-Amino-N-(4-(1-methyl-1 H-be nzo[d]imi dazol-2-yl) -3-(methylthi o)-1 -phenyl-1 H-pyr azol-5-yl)-1,5-di phenyl1H-pyrazole-4-carboxami de (25b)

Pale orange crystals, $\left(48 \%\right.$, yie ld), mp $297-300^{\circ} \mathrm{C}$; IR $v_{\max }$ $/ \mathrm{cm}^{-1}(\mathrm{KBr}) 3368\left(\mathrm{NH}_{2}\right), 3262(\mathrm{NH}), 1648(\mathrm{CO}), 1625$ $(\mathrm{C}=\mathrm{N}) ;{ }^{1} \mathrm{H}$ NMR $\left(\mathrm{DMSO}_{6}\right) \delta 2.70\left(\mathrm{~s}, 3 \mathrm{H}, \mathrm{SCH}_{3}\right), 3.89(\mathrm{~s}$, $\left.3 \mathrm{H}, \mathrm{NCH}_{3}\right), 4.11\left(\mathrm{~s}, 2 \mathrm{H}, \mathrm{NH}_{2}\right), 7.31-7.81(\mathrm{~m}, 19 \mathrm{H}, \mathrm{Ar}-\mathrm{H})$, $10.29(\mathrm{~s}, 1 \mathrm{H}, \mathrm{NH}) ; \mathrm{m} / \mathrm{z} 596\left(\mathrm{M}^{+}, 19.83\right)$. Anal. Calcd. For $\mathrm{C}_{34} \mathrm{H}_{28} \mathrm{~N}_{8} \mathrm{OS}$ (596.7): C, 68.44; H, 4.73; N, 18.78; S, 5.30\%. Found: C, 68.42; H, 4.70; N, 18.77; S, 5.21\%.

3).N-(4-Benzyli dene-4,5-dih ydr o-5-amin o-1 H-pyr azol3-yl)-4-(1-me thyl-1 H-benzo-[d]i mi dazol-2-yl)-3-(methylt hio)-1-phenyl-1 H-pyrazol-5-ami ne (26)

yellow crystals, (13\%, yield), mp $129-131^{\circ} \mathrm{C}$; IR $v_{\max } /$ $\mathrm{cm}^{-1}(\mathrm{KBr}) 3248(\mathrm{NH}), 1629(\mathrm{C}=\mathrm{N}) . \mathrm{m} / \mathrm{z} 504\left(\mathrm{M}^{+}, 32.54\right)$. Anal. Calcd. For $\mathrm{C}_{28} \mathrm{H}_{24} \mathrm{~N}_{8} \mathrm{~S}$ (504.61): C, 66.65; H, 4.79; N, 22.21 ; S, 6.35\%. Found: C, 66.61; H, 4.78; N, 22.19; S, $6.36 \%$.

\section{REFERNCES}

[1] Liu, X. H.; Cui, P.; Song, B. A.; Bhadury, P. S.; Zhu, H. L.; Wang, S. F. Bioorg. Med. Chem. 2008, 16, 4075.

[2] Ouyang, G.; Chen, Z.; Cai, X. J.; Song, B. A.; Bhadury, P. S.; Yang, S.; Jin, L. H.; Xue, W,; Hu, D. Y.; Zeng, S. Bioorg. Med. Chem. 2008, 16, 9699

[3] Abdel-Hafez, E. M. N.; Rahma, G. E. A. A.; Aziz, M. A.; Radwan, M. F.; Farag, H. H. Bioorg. Med. Chem. 2009, 17, 3829 .

[4] Park, H. J.; Lee, K.; Park, S. J.; Ahn, B.; Lee, J. C.; Cho, H. Y.; Lee, K. I. Bioorg. Med. Chem. Lett. 2005, 15, 3307.

[5] Ouyang, G.; Cai, X. J.; Chen, Z.; Song, B. A.; Bhadury, P. S.; Yang, S.; Jin, L. H.; Xue, W,; Hu, D. Y.; Zeng, S. J. Agric. Food Chem. 2008, 56, 101601.

[6] Dai, H.; Li, Y. Q.; Du, D.; Qin, X.; Zhang, X.; Yu, H. B.; Fang, J. X. Food Chem. 2008, 56, 10805.

[7] Riyadh, S. M.; Farghaly, T. A.; Abdallah, M. A.; Abdalla, M. M.; El-Aziz, M. R. A. Eur. J. Med. Chem. 2010, 45, 1042.

[8] Anzaldi, M.; Maccio, C.; Mazzei, M.; Bertolotto, M.; Ottonello, L.; Dallegri, F.; Balbi, A. Chem. Biodivers, 2009, 6, 1674.

[9] El-Shafei, A.; Fadda, A. A.; Khalil, A. M.; Ameen, T. A. E.; Badria, F. A. Bioorg. Med. Chem. 2009, 17, 5096.

[10] Nassar, E. J. Am. Sci. 2010, 6, 463.

[11] Azarifar, D.; Shaebanzadeh, M. Molecules, 2002, 7, 885.

[12] Ali, M. A.; Siddiqui, A. A.; Shahayar, M. Eur. J. Chem. 2007, 
42,268

[13] Amir, M.; Kumar, H.; Khan, S. A. Bioorg. Med. Chem Lett. 2008, 18, 918.

[14] Sharshira, E. M.; Hamada, N. M. M. Molecules, 2011, 16, 7736.

[15] Hamada, N. M. M; Sharshira, E. M. Molecules, 2011, 16, 2304.

[16] Kalirajan, R.; Sivakumar, S. U.; Jubie, S.; Gowramma, B.; Suresh, B. Int. J. Chem. Tech. Res. 2009, 1, 27.

[17] Palaska, E.; Erol, D.; Demirdamar, R. Eur. J. Med., Chem., 1996, 31, 43.

[18] Palaska, E.; Aytemir, M.; Uzbay, I. T.; Erol, D.; Eur. J. Med. Chem., 2001, 36, 539.

[19] Dmytro, H.; Borys, Z.; Olexandr, V.;Lucjusz Z.;Andrzej, G.;Roman, L. Eur. j. Med. Chem. 2009, 44, 1396.

[20] Bilgin, A. A.; Palaska, E.; Sunal, R.; Drug Res. 1993, 43, 1041.

[21] Mui, M. S.; Siew, B. N.; Buss, A. D.; Crasta, S. C.; Kah, L. G.; Sue, K. L. Bioorg. Med. Chem. Lett. 2002, 12, 679.

[22] Parmar, S. S.; Pandey, B. R.; Dwivedi, C.; Harbison, R. D. J. Pharm. Sci. 1974, 63, 1152.

[23] Siddiqui, A. A.; Rahman, M. A.; Shaharyar, M.; Mishra, R. Chem. Sci. J. 2010, 8, 1.

[24] Soni, N.; Pande, K.; Kalsi, R.; Gupta, T. K.; Paemar, S. S.; Barthwal, J. B. Res. Commun. Pathol. Pharmacol. 1987, 56, 129.

[25] Ragavan, R. V.; Vijayakumar, V.; Kumari, N. S. Bioorg. Med. Chem. 2001, 9, 2149, 45(3), 1173.

[26] Ali, E. T.; El.kazak, A. M. Eur. J. Chem. 2010, 1(1), 6.

[27] Karegoudar, P.; Karthikey an, M.S.; Prasad, D. J.; Mahalinga, M.; Holla, B. S.; Kumari, N. S. Eur. J. Med. Chem. 2008, 43, 261.

[28] Vicini, P.; Geronkiaki, A.; Incerti, M.; Zani, F.; Dearden, J.; Hewitt, M. Bioorg. Med. Chem. 2008, 16, 3714.

[29] Basavaraja, K. M.; Somasekhar, B.; Appalaraju, S. Ind. J. Heterocycl. Chem. 2008, 18, 69.

[30] Karabasanagouda, T.; Adhikari, A. V.; Ramgopal, D.; Parameshwarappa, G. Ind. J. Chem. 2008, 47B, 144.

[31] Amine, M. A. K.; Abdel Rahman, D. E.; El-Eryani, Y. A. Bioorg. Med. Chem. 2008, 16, 5377.

[32] Andreani, A.; Rambaldi, M.; Leoni, A.; Locatelli, A.; Bossa, R.; Chiericozzi, M.; Galatulas, I.; Salvatore, G. Eur. J. Med. Chem. 1996, 31, 383.

[33] Jiang, X.; Gu, H. Bioorg. Med. Chem. 2000, 8, 363.

[34] Abbs, T. F.; Reji, F.; Devi, S. K. C.; Thomas, K. K.; Sreejalekshmi, K. G.; M anju, S. L.; Francis, M.; Philip, S. K.; Bharathan, A.; Rajasekharan, K. N. Ind. J. Chem. 2008, 47B, 1145.

[35] Chowki D; Magdum, C. S.; Ladda, P. L.; Mohite, S. K. Int. J. Chem. Sci. 2008, 6(3), 1600.
[36] Bhusari, K. P.; Khedekar, P. W.; Umathe, S. N.; Bahekar, R. H.; Raghu, R. R. A. Ind. J. Heterocycl. Chem. 2000, 9, 275.

[37] Taori, K.; Paul, J. V.; Luesch, H. J. Am. Chem. Soc. 2008, 130, 1806.

[38] Chakrabarti, J. K.; Horsman, L.; Hotten, T. M.; Pullar, I. A.; Tupper, D. E.; Wright, F. C. J. Med. Chem. 1980, 23, 878.

[39] Ram, V. J.; Pandey, H. K.; Vlientick, A. J. J. Heterocycl. Chem. 1981, 18, 1277.

[40] Evans, B. E.; Rittle, K. E.; Bock, M. G.; DiPardo, R. M.; Freidin ger, R. M.; Whitter, W. L.; Lundell, G. F.; Veber, D. F.; Anderson, P. S.; Change, R. SSS. L.; Lotti, V. J.; Cerino, D. J.; Chen, T. B.; Kling, P. J.; Kunkel, K. A.; Springer, J. P.; Hirshfield, J. J. Med. Chem. 1988, 31, 2235.

[41] Horton, D. A.; Bourne, G. T.; Smy the, M. L. Chem. Rev. 2003, 103, 893 .

[42] (a) Labanauskas, L.; Kalcas, V.; Udrenaite, E.; Gaidelis, P.; Brukstus, A.; Dauksas, V.; Pharmazie 2001, 56, 617. (b) Schenone, S.; Bruno, O.; Ranise, A.; Bondavalli, F.; Fillippelli, W.; Falcone, G.; Giordano, L.; Vitelli, M. R. Bioorg. Med. Chem. 2001, 9, 2149.

[43] Dogan, H. N.; Duran, A.; Rollas, S.; Sener, G.; Uysat, M. K.; Gulen, D. Bioorg. Med. Chem. 2002, 10, 2893.

[44] (a) Ilies, M. A.; Masereel, B.; Rolin, S.; Scozzafava, A.; Campeanu, G.; Cimpeanu, V.; Supuran, C. T. Bioorg. Med. Chem. 2004, 12, 2717. (b) Archana; A.; Srivastava, V. K.; Kumar, A. Eur. J. Med. Chem. 2002, 37, 873.

[45] Mohareb, R. M.; EL-sayed, N. N. E.; Abdelaziz, M. A. Molecules, 2012, 17, 8449.

[46] Metwally, M. A.; Keshk, E. M.; Fekry, A.; Etman, H. A. J. Chem. Res. 2004, 3, 602.

[47] Bhawal, B. M.; Khanapure, S. P.; Biehl, E. R. Synth. Commun. 1990, 20, 3235.

[48] Fadda, A. A.; Mukhtar, M. M.; Refat, H. M. Am. J. Org. Chem. 2012, 2(2), 32.[refs. cited in]

[49] Elnagdi, M. H.; Sadek, K. U.; Elmaghraby, M. A.; Selim, M. A.; Khalafallah, A. K.; Raslan, M. A. Phosphorus, Sulfur, and Silicon 1995, 105, 51.

[50] Sayed, S. M.; Raslan, M. A.; Khalil. M. A.; Dawood, K. M. Heteroatom Chem. 1999, 10(5), 385.

[51] Raslan, M. A.; Sayed, S. M.; Khalil. M. A.; Farag, A. M. Heteroatom Chem. 2000, 11(2), 94.

[52] Sayed, S. M.; Selim, M. A.; Raslan, M. A.; Khalil. M. A. Heteroatom Chem. 2000, 11(5), 362.

[53] Sayed, S. M.; Khalil. M. A.; Selim, M. A; Raslan, M. A. Synth. Commun. 2002, 32(2), 481.

[54] Raslan, M. A.; Khalil. M. A. Heteroatom Chem. 2003, 14(2), 114.

[55] Dawood, K. M.; Raslan, M. A.; Farag, A. M. Synth. Commun. 2003, 33(23), 4079.

[56] Raslan, M. A.; Abd el-aal, R. M.; Hassan, M. E.; Ahmed, N. A.; Sadek, K. U. J. Chin. Chem. Soc. 2001, 48, 91.

[57] Dawood, K. M.; Raslan, M. A. J. Heterocycl. Chem. 2008, 45, 
170 Mohamed A. Khalil et al:: Reactivity of 2-Cyano-N-(4-(1-Methyl-1H-benzo[d]imidazol-2-yl)-3-(M ethylthio)-1-Pheny l-1HPyrazol-5-yl)Acetamide: A Facile Synthesis of Pyrazole, Thiazole, 1,3,4-Thiadiazole and Poly substituted Thiophene Derivatives

245.

[58] Raslan, M. A.; Abd el latif, F. M.; Otto, H. H.; Sadek, K. U. Org. Prep. Proc. Int. 2000, 32(3), 276.

[59] Mohareb, R. M.; Aziz, S. I.; Sayed, N. I. A.; Shams, H. Z. J. Chin. Chem. Soc. 1992, 39, 181.

[60] Ried, W.; Schleimer, B. Angew. Chem. 1968, 70, 164; Chem.
Abstr. 1959, 53, $1314 \mathrm{f}$.

61] Farag, A. M. Org. Prep. Proc. Int. 1988, 18, 285.

[62] Wolkoff, P. Canadian J. Chem. 1975, 53, 333.

[63] Shawali, A. S.; Osman, A. Tetrahedron, 1971, 27, 2517.

[64] Sayed, S. M.; Khalil, M. A.; Raslan, M. A. American J. ofOrg. Chem. 2012, 2(6). 